\title{
Dysfunctional and compensatory duality in mild cognitive impairment during a continuous recognition memory task
}

\author{
Sara Aurtenetxe , Nazareth P. Castellanos ， Stephan Moratti , Ricardo Bajo , Pedro Gil , \\ Garikoitz Beitia , Francisco del-Pozo , Fernando Maestú
}

\begin{abstract}
One of the current issues of debate in the study of mild cognitive impairment (MCI) is deviations of oscillatory brain responses from normal brain states and its dynamics. This work aims to characterize the differences of power in brain oscillations during the execution of a recognition memory task in MCI subjects in comparison with elderly controls. Magnetoencephalographic (MEG) signals were recorded during a continuous recognition memory task performance. Oscillatory brain activity during the recognition phase of the task was analyzed by wavelet transform in the source space by means of minimum norm algorithm. Both groups obtained a $77 \%$ hit ratio. In comparison with healthy controls, $\mathrm{MCl}$ subjects showed increased theta $(\mathrm{p}<0.001)$, lower beta reduction $(p<0.001)$ and decreased alpha and gamma power $(p<0.002$ and $p<0.001$ respectively $)$ in frontal, temporal and parietal areas during early and late latencies. Our results point towards a dual pattern of activity (increase and decrease) which is indicative of $\mathrm{MCl}$ and specific to certain time windows, frequency bands and brain regions. These results could represent two neurophysiological sides of MCI. Characterizing these opposing processes may contribute to the understanding of the disorder.
\end{abstract}

\section{Introduction}

Mild cognitive impairment (MCI) has been considered as a major precursor of Alzheimer's disease (AD). Evidence suggests that the conversion rate from $\mathrm{MCI}$ to dementia is of about $12 \%$ per year, while healthy controls convert at a $1-2 \%$ rate, and that $\mathrm{MCl}$ show a clear cognitive profile and neuropathological signs of AD (Petersen, 2004; Petersen et al., 2001, 2006; see Twamley et al. (2006) for a review of neuropsychological and neuroimaging literature on preclinical AD). Thus, early identification of patients at risk for the development of dementia, such as $\mathrm{MCI}$ population, might be crucial for providing them cognitive and/or pharmacological interventions to slow down the progression of cognitive deficits and retard the onset of disability.

It has been consistently demonstrated that brain oscillations underlie human information processing and fundamental aspects of cognition (Buzaki, 2006). Since memory processes have been shown to be related to oscillations in the theta, alpha, beta, and gamma frequency ranges (Klimesch, 1999; Tallon-Baudry et al., 1999; Jensen and Tesche, 2002; Leiberg et al., 2006), spectral changes during such tasks are good candidates to study memory related pathologies.

Memory loss is the most common clinical symptom of $\mathrm{MCI}$ and $\mathrm{AD}$ (Baddeley, 1991; Belleville et al., 1996; Alescio-Lautier et al., 2007; Caza and Belleville, 2008). fMRI neuroimaging studies have extensively shown brain activity differences between $\mathrm{MCI}$ and controls (Clément and Belleville, 2010; for a review see Dickerson and Sperling, 2008). Although the majority of the MEG and EEG research in $\mathrm{AD}$ and $\mathrm{MCI}$ have focused on resting state recordings, the dynamics of the neuronal electromagnetic activity during a memory task could provide more reliable information to study cognitive consequences of pathological aging (Van der Hiele et al., 2007a; Giannakopoulos et al., 2009). In this line, EEG and MEG studies of modified versions of the Sternberg letter-probe task (De Toledo-Morrell et al., 1991) have been able to address patterns of brain activity specific to $\mathrm{MCl}$ pathology (Hogan et al., 2003; Maestú et al., 2008; Bajo et al., 2010). These studies are based on event related field source reconstruction analysis and on fast-Fourier transform, time-frequency and connectivity analysis in sensor space, respectively. Therefore, the study of the time-frequency MEG dynamics in source space during the recognition stage of a memory task has not been addressed yet in $\mathrm{MCl}$ patients (see below the main 
statements of the present study). Two previous studies evaluated time-frequency activity in $\mathrm{MCI}$ patients using the Sternberg paradigm (Karrasch et al., 2006; Kurimoto et al., 2011). These studies analyzed the encoding and/or maintenance stages of the task. Only one of them found differences between controls and MCls and these differences were found in the sensor space. Thus, it seems necessary a study evaluating: 1) time-frequency power; 2) at the source space; 3 ) at the recognition stage of a memory task.

Electroencephalographic brain activity modulations have been variably reported in the characterization of $\mathrm{MCl}$ (Grunwald et al., 2002; Jiang, 2005; Missonnier et al., 2006a; Van der Hiele et al., 2007a,b; Zheng et al., 2007; Cummins et al., 2008; Deiber et al., 2009). In this line, fMRI literature of cognitive aging postulates that activity increases are associated with neural compensation by means of additional recruitment of neurons in order to prevent performance reductions (Cabeza et al., 2002; Dickerson et al., 2005; Grady et al., 2005). On the contrary, activity decreases have been related to brain dysfunction and neuronal loss as a degenerative process causing reduction in accuracy (Friston and Price, 2003; Scarmeas et al., 2003; Johnson et al., 2012). Overall, activity decrease may be a precursor of AD and would need a follow-up for verification. Thus, the variety of results reported so far could reflect pathological compensatory and dysfunctional processes acting simultaneously in the altered brain (Clément and Belleville, 2010).

In order to study task-related brain dynamics of $\mathrm{MCI}$, we report brain spatial-time-frequency differences between $\mathrm{MCl}$ patients and controls during a continuous recognition memory task based on the following statements: 1) the data will be recorded during the execution of a memory task because differences between groups will be highlighted (Van der Hiele et al., 2007a); 2) data will be acquired with MEG due to its high temporal resolution; 3) a time-frequency analysis (with a wavelet transform, WT, approach) will be applied and offer the opportunity to study differential dynamics of the activity across the frequency spectrum; 4) data will be analyzed in the source space, which will provide an accurate information about the brain regions involved; 5) the patterns observed in the control group will be the gold standard pattern of activation. Thus, any deviation in brain activity from controls (both hyper and hypoactivation) will be considered pathological.

Compensatory activity will be considered when a particular profile of activation (increasing or decreasing respect to the control group) demonstrates an improvement in cognitive function (i.e. positive correlation between brain activation at a certain frequency band and performance on memory test). Dysfunctional activity will be considered when an activity pattern deviates from the control group without any sign of improving cognitive function. This analysis will offer a further step in the knowledge of the brain dynamics underlying $\mathrm{MCl}$, and will allow us to evaluate the compensatory and dysfunctional hypothesis.

\section{Materials and methods}

\subsection{Subjects}

Twenty-five age matched elderly subjects (see Table 1) participated in the study. Participants were divided into two groups based on their clinical profiles: 12 were diagnosed with amnesic MCI and 13 were considered as elderly healthy control subjects. Patients were recruited from the Geriatric Unit of the University Hospital San Carlos (Madrid, Spain), whereas controls were recruited from a day care center. To avoid potential sources of differences due to handedness and its relation with hemispheric dominance that could affect the organization of brain activity, we decided to keep this variable stable across subjects (see Long et al., 2012). Thus, participants (patients and controls) were all right handed ( $>40$ in the Oldfield's Edinburgh Handedness Inventory (Oldfield, 1971); following the formula $R=(D / C T) \times 100$; where $D$ is right handed $(\mathrm{RH})-$ left handed $(\mathrm{LH})$ and $\mathrm{CT}$ is $\mathrm{LH}+\mathrm{RH})$.

$\mathrm{MCl}$ diagnosis was established according to the criteria proposed by Petersen (2004). Thus, MCI patients fulfilled the following criteria: (1) cognitive complaint corroborated by an informant (a person who stays with the patient for half a day almost 4 days a week); (2) objective cognitive impairment, documented by delayed recall from the Logical Memory II subtest of the Wechsler Memory Scale Revised (cut-off scores $\leq 16$ for $\geq 16$ years of education; $\leq 8$ for $\geq 8$-15 years of education) (MCI's scores: Logical Memory I (LM-I) $=10.3 \pm 3.9$; Logical Memory-II (LM-II) $=3 \pm 3.5$ ); (3) normal general cognitive function, as determined by clinician's judgment based on a structured interview with the patient and an informant. Additionally a MMSE score greater than $24 ;(4)$ preserved daily living activities (assessed with the Spanish version of the Functional Assessment Scale (Pfeffer et al., 1982)); (5) not sufficiently impaired, cognitively and functionally to meet criteria for dementia. According to their clinical and neuropsychological profiles all subjects in this group were considered amnesic MCI.

The healthy control group was selected according to a neuropsychological assessment and based on the demographic characteristics of the experimental group. Exclusion criteria for the selection of all participants included (1) previous medical history of psychiatric or any other neurological disease; (2) psychoactive drugs consumption and (3) severe sensory or comprehension deficits. Before the MEG recording, all participants or legal representatives signed an informed consent that explains the technical and ethical considerations of the technique. The Local Ethics Committee had approved the study.

\subsection{Stimuli and task}

MEG scans were obtained in the context of a modified version of the Sternberg's letter-probe task (De Toledo-Morrell et al., 1991; Maestú et al., 2001). During the task a set of five letters was presented simultaneously for $30 \mathrm{~s}$ and the subjects were asked to keep the letters in mind (encoding phase). After the presentation of the fiveletter set, a series of single letters $(500 \mathrm{~ms}$ in duration with a random interval between 2 and $3 \mathrm{~s}$ ) was introduced one at a time, and the participants were asked to press a button with their right hand when a letter of the previous set was detected (recognition phase). The list consisted of 250 letters. Half of them were targets (previously presented letters), and the remaining letters were distractors (not previously presented letters). All of the subjects practiced a training series before the real test, and the single letter presentation did not start until the participant demonstrated that she/he remembered the five-letter set. Letters were projected by an LCD video-projector (SONY VPL-X600E) located outside the magnetically shielded-room onto a series of in-room mirrors, the last of which was suspended approximately $1 \mathrm{~m}$ above the subject's face. The letters subtended $1.8^{\circ}$ and $3^{\circ}$ of the horizontal and vertical visual angle, respectively.

Table 1

Demographic characteristics and cognitive data for the control and MCI groups.

\begin{tabular}{|c|c|c|c|c|c|c|c|}
\hline & Age & MMSE & GDS & RT (ms) & Hits & Errors & $\mathrm{CR}$ \\
\hline CNT & $70.62 \pm 6.3$ & $28.7 \pm 1.4$ & 1 & $659 \pm 74$ & $97 \pm 23.5$ & $10.1 \pm 12.9$ & $114.8 \pm 12.9$ \\
\hline $\mathrm{MCI}$ & $74.83 \pm 4.8$ & $25.3 \pm 1.8$ & 3 & $631 \pm 72$ & $97 \pm 29.4$ & $14.8 \pm 25.6$ & $106.6 \pm 25.4$ \\
\hline
\end{tabular}

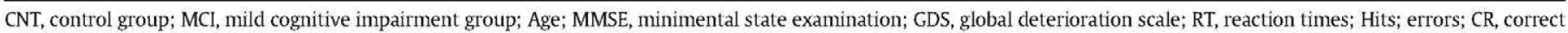
rejections. 


\subsection{MEG data collection}

The MEG signal was recorded using a 148-channel whole-head magnetometer (MAGNES (B) 2500, 4-D Neuroimaging Inc., San Diego, CA, United States) situated in a magnetically shielded room (see Fig. 1 for schematic illustration of experimental and analysis protocol). The sampling rate was $254.31 \mathrm{~Hz}$. The signal was filtered online with a band pass filter between 0.1 and $50 \mathrm{~Hz}$. Then, the data were submitted to a noise reduction procedure, which uses simultaneous recordings from nine gradiometer reference channels that are part of the MEG system. Due to a high percentage of correct responses (77\% of hits, correct responses, in both groups) and to a low percentage of errors ( $10 \%$ of errors in the control group and $15 \%$ of errors in the $\mathrm{MCl}$ group), our analysis was based on the recognition phase of the target stimuli to ensure equal signal to noise ratios across groups. Epochs (composed of $3000 \mathrm{~ms}$; from $1500 \mathrm{~ms}$ before to $1500 \mathrm{~ms}$ after the stimulus onset) contaminated by ocular artefacts were corrected by means of BESA artefact-correction tool (Brain Electrical Source Analysis). Trials containing muscular artefacts were discarded from analysis. Overall, a minimum of 80 artefact-free epochs (64\%) was entered in the analysis.

\subsection{Wavelet spectrum and source estimation}

Time-frequency (TF) representation of MEG data was calculated on a single trial basis for a $3000 \mathrm{~ms}$ time window. Data was based on the wavelet transform of the signals (Tallon-Baudry et al., 1997; Mallat, 1998) using a Morlet wavelet function with a width of 5 cycles per wavelet in a spectral range between 2 and $88 \mathrm{~Hz}$, in $1 \mathrm{~Hz}$ steps. Wavelet transform is a dynamical alternative to Fourier, used to perform time spectral analyses for non-stationary time series. The continuous wavelet transform of a signal suppose its projection onto a set of basic functions obtained from mother wavelet by rescaling and translating it along the time axis. Wavelet coefficients of the signal in the time-spectral plane were obtained. TF representation was estimated using custom-written scripts in MATLAB Version 7.4 (Mathworks, Natick, MA).

Before estimating the cortical generators of the power changes at different latencies and frequency bands, the head sensor positions of each subject were spatially aligned (co-registered) with a surface brain model (3003 surface dipoles). The dipole mesh (representing sulci and gyri) was derived from a Collin 27 template brain aligned with the Montreal Neurological Institute (MNI) phantom brain as implemented in the Fieldtrip software package (http://www.ru.nl/ fcdonders/fieldtrip/) (Collins et al., 1998). Thereby, in a first step the template brain's fiducials and the individually digitized fiducials were realigned. In a second step, subject specific digitized head shape points were fitted to the template scalp surface by minimizing the mean distance between the individual head shape points and the template scalp surface. After the co-registration of the MEG and phantom brain coordinate systems, the forward solution was calculated to determine the lead field matrix for further source inversion, using a head model based on overlapping spheres. For each channel a local sphere was fitted to the underlying head shape points (Huang et al., 1999).

The minimum-norm estimation procedure (MNE) was used to perform the source localization of the TF MEG signals. The underlying generator sources were estimated from wavelet coefficients (by combining the source projected real and imaginary parts) using the Fieldtrip software package (http://www.ru.nl/fcdonders/fieldtrip/) and custom-written scripts. Jensen and Vanni (2002) demonstrated that by transforming the real and imaginary parts of the Fourier components in the source domain by means of MNE, it is possible to identify source areas of rhythmic activity in the frequency domain. Source representation of the time-frequency data was constructed by means of an L2 MNE procedure in the frequency domain, with a standard Tikhonov regularization to control the noise of the data (Bouhamidi and Jbilou, 2007). Accordingly, in our study the underlying current source density (the source strength at each node of the MNI phantom brain) of four frequency bands (theta $4-8 \mathrm{~Hz}$; alpha, $8-12 \mathrm{~Hz}$; beta, $12-30 \mathrm{~Hz}$; gamma, $30-45 \mathrm{~Hz}$ ) was estimated (Jensen and Vanni, 2002; Moratti et al., 2008). Note that the subdivision of the frequency bands in sub-bands (i.e. low/high alpha and beta bands) can offer information about specific cognitive processes such as attention, task specific or motor related processes (Neuper and Pfurtscheller, 2001; Klimesch et al., 1998). However, an analysis of the sub-bands did not reveal differences when considering high and low alpha or beta bands separately. Therefore, we decided to maintain the classic divisions of the frequency bands in order to offer a holistic characterization of the spectrum between healthy controls and patients.

For the estimation of the underlying current source density, the real and imaginary parts of each wavelet component averaged within each of the four frequency bands was submitted to the MNE analysis. Thereafter, the MNE of the real and imaginary parts was combined by using the root square of the sum of squares of the two wavelet parts as an estimate of absolute amplitude. The change in amplitude was calculated with respect to a baseline period before the beginning of each epoch. For each frequency band, the mean time-frequency amplitude of the prestimulus period (between 1000 and 0 ms before stimulus onset) was considered as a baseline and subtracted from the time-frequency representation in order to normalize it. Finally, data were log-transformed in order to

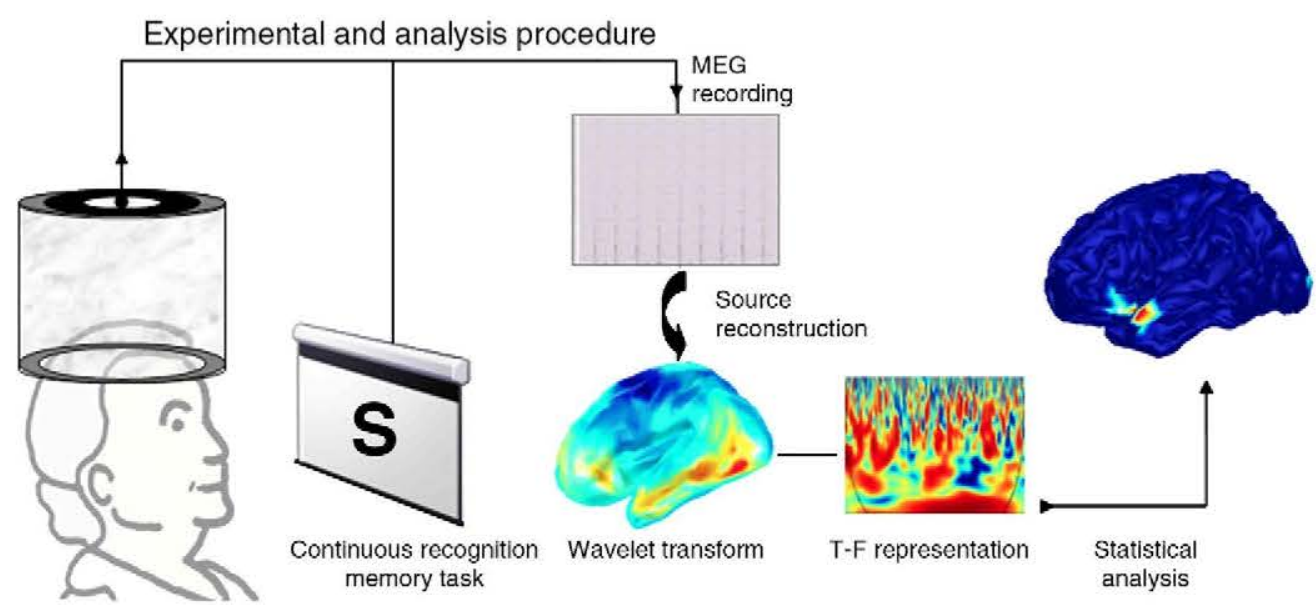

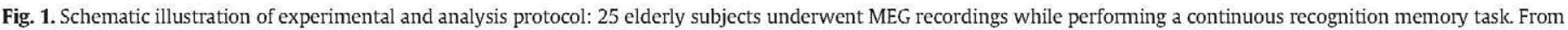

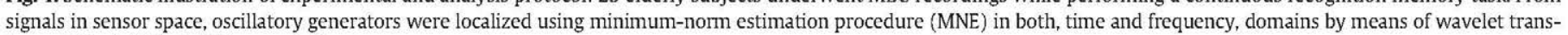
form. Only statistical differences between groups are shown. 
reduce the effect of the non-Gaussian distribution (Pivik et al., 1993). As MEG is more sensitive to tangential sources with respect to the scalp surface, source activity is often localized within the sulci of the neo-cortex. For better visibility of this activity, MNE results are shown on a smoothed MNI brain surface.

\subsection{Statistical analysis}

Before the estimation of the source activity, a control analysis was performed to test possible baseline differences between groups. Results showed no significant differences between groups at baseline (all comparisons show $\mathrm{p}>0.05$ ), which indicates that differences observed in the post-stimulus interval are not influenced by differences in baseline.

A baseline corrected segment of $1000 \mathrm{~ms}$ after target stimulus onset was considered for statistical analysis. Source activity in the timefrequency domain was averaged over 26 time-windows of $39 \mathrm{~ms}$ each. Power values of each participant and at each dipole location of the brain surface mesh were statistically analyzed by using a Kruskall Wallis test to compare the spatial-time-frequency patterns between the patient and the healthy control group. Associated p-values were thresholded at $\mathrm{p}<0.002$ (uncorrected) (see Gurtubay et al., 2001; Brookes et al., 2005; Osipova et al., 2005; Stam et al., 2006; Maestú et al., 2008; for a similar statistical approach). In addition, in order to reinforce the robustness of the results, a clustering analysis was applied (at least 2 neighbor brain sources or time points had to be significant at a p level of 0.002 ). The spatial and temporal clustering required, enhances the consistency of the data and strengthens its cohesion by avoiding spurious spatial changes in isolated sources and spurious dynamical fluctuations across time. Further post-hoc analysis was performed to explore whether age, task performance and/or neuropsychological test scores of $\mathrm{MCI}$ and control subjects were related to the power changes at statistically significant source clusters and time windows (according to the previously calculated statistical differences between groups per frequency band). Subsequently, Spearman's correlation coefficients were calculated with a cut-off of $p<0.03$.

\section{Results}

\subsection{Behavioral performance}

Behavioral performance during the continuous recognition memory task showed: hits (77\%), errors (8\%) and correct rejections (92\%) in the control group. Performance in the MCI group was as follows: hits (77\%), errors (12\%) and correct rejections (85\%).

These results revealed no significant differences between groups, neither with respect to the number of hits $[t(23)=0.007, p=0.994]$, nor to the number of errors $[t(23)=0.584 ; p=0.565]$ or correct $[t(23)=1.025, p=0.316]$ rejections. The percentage of hits $(77 \%$ for the control group and $77 \%$ for the $\mathrm{MCl}$ group) and correct rejections ( $92 \%$ control group and $85 \%$ MCI group) was high enough in both groups to indicate that all participants were committed to the task. Reaction times did not show significant differences between groups $[\mathrm{t}(23)=0.871, \mathrm{p}=0.395]$.

\subsection{Spectral power dynamics in source space}

Time-Frequency source representation for target stimuli was compared between $\mathrm{MCI}$ and healthy control participants. Statistical differences between $\mathrm{MCI}$ subjects and controls are summarized below (Fig. 2).

\subsubsection{Theta spectral band}

Compared to healthy controls, $\mathrm{MCI}$ patients showed higher induced theta power during the recognition phase in the right frontal pole $(\mathrm{p}<0.001)$ between 99 and $294 \mathrm{~ms}$.

\subsubsection{Alpha spectral band}

However, between 489 and 528 ms after stimulus onset, MCI subjects were characterized by lower alpha power values in right temporooccipital cortex $(\mathrm{p}<0.002)$.

\subsubsection{Beta spectral band}

Differences in beta power were localized in bilateral posterior parietal cortex and in parieto-temporal, frontal lateral and temporal posterior superior areas of the right hemisphere between 645 and $801 \mathrm{~ms}(\mathrm{p}<0.001)$. The results indicate higher power values for the $\mathrm{MCI}$ group in comparison with healthy controls.

\subsubsection{Gamma spectral band}

Compared to controls, $\mathrm{MCI}$ patients demonstrated lower gamma power in left posterior parietal cortex between 372 and $411 \mathrm{~ms}$ and between 450 and $489 \mathrm{~ms}(\mathrm{p}<0.001)$.

\subsection{Correlation analysis}

Post-hoc analysis of the statistical differences in power and age, task performance and test scores between groups revealed linear relationships between theta oscillations and LM-I subtest $(r=0.692$, $\mathrm{p}<0.03$ ) for MCI subjects (see Fig. 3).

\section{Discussion}

This study aimed at characterizing brain oscillatory power profiles of MCI patients, compared with healthy controls, at cortical source level during a continuous recognition memory task. Our neurophysiological results showed higher theta power and less beta power reduction in $\mathrm{MCI}$ subjects compared to controls. However, $\mathrm{MCl}$ patients were also characterized by reduced alpha and gamma band power. The positive correlation obtained between theta oscillatory activity and performance on the memory test, while others did not achieve any correlation and/or showed a diminution of activity in comparison with the control group, leads us to suggest the presence of a brain duality pattern in the MCI brain. The fact that both groups achieved a high percentage of hits indicates an adequate sustained attention while performing the task and allows us to compare data more readily. Present findings offer a new perspective about the features and changes of a MCI brain, and contribute to the knowledge of the continuum to $\mathrm{AD}$. Our results, in addition to previous literature, point out the capacity of current modified Sternberg task to generate specific brain activity patterns which help to differentiate between clinical profiles.

The group with MCI showed an increase in theta power over the right frontal pole at early latencies (99-294 ms) in comparison to the control group. The fact that this increased activity achieved a positive correlation with the performance on memory test (see Fig. 3) allowed us to interpret this activity as compensatory due to its relation with an improvement in the recall of information. Thus, cognitively more preserved MCIs (those who obtained higher scores in the LM-I subtest) were those who showed higher theta relative power compared to controls, and vice versa, suggesting a possible relationship between theta power and cognitive status in $\mathrm{MCI}$ subjects. Classical studies with EEG (Klimesch, 1996) demonstrate that during memory tasks theta activity tends to increase and correlate with performance. In fact, the coupling in theta between different regions of the medial temporal lobe has been correlated with improving task execution on memory task (Fell et al., 2003; Axmacher et al., 2010). In line with our study Finnigan and Robertson (2011) observed positive correlations between theta and cognitive performance. Further during haptic tasks, theta power increases have been suitable measures to distinguish healthy subjects from subjects with $\mathrm{MCI}$ and mild dementia (Grunwald et al., 2002). In a similar manner, fMRI studies show higher brain activity in MCI participants that in healthy controls 
THETA

$\mathrm{MCl}>\mathrm{CNT}$

$\mathrm{p}<0.001$

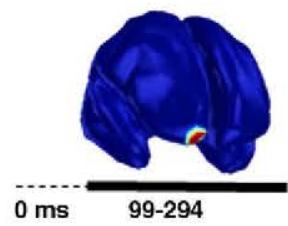

ALPHA

$\mathrm{MCl}<\mathrm{CNT}$

$\mathrm{p}<0.002$

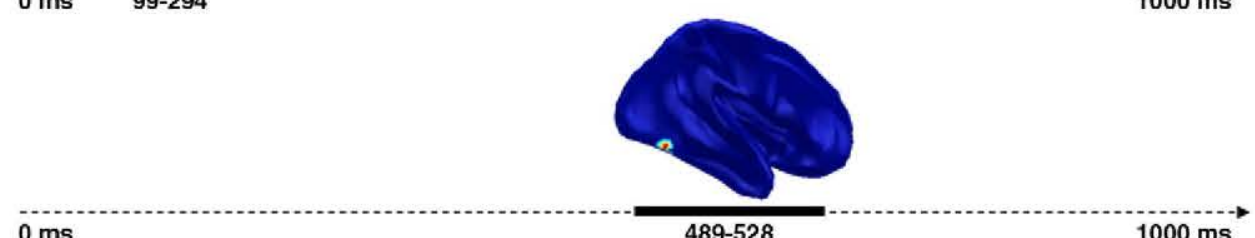

$0 \mathrm{~ms} \quad 489-528 \quad 1000 \mathrm{~ms}$

BETA

$\mathrm{MCl}>\mathrm{CNT}$

$p<0.001$

$\mathrm{MCl}<\mathrm{CNT}$

$p<0.001$

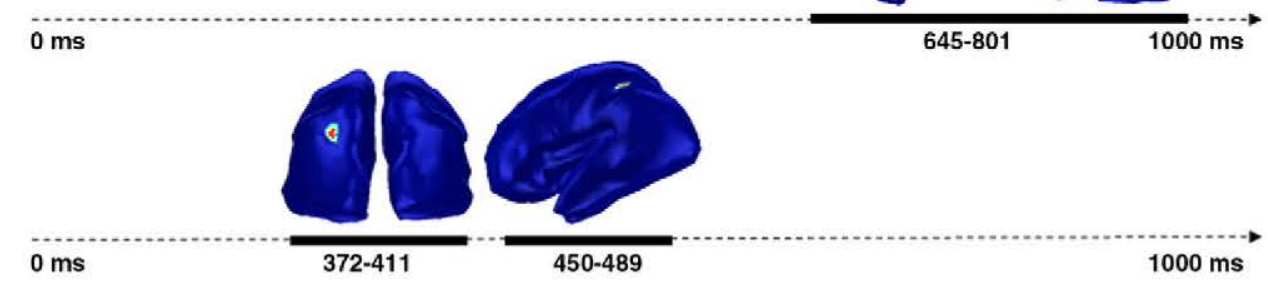

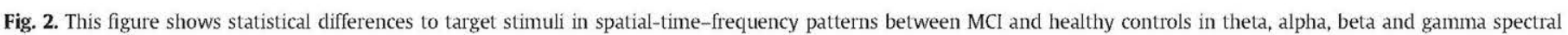
bands. Frequency band, group differences and corresponding p values are shown in left. A time axis is included to remark when differences occur.

during memory tasks (Bokde et al., 2010), which tends to reduce when the memory load increases (Kochan et al., 2010).

Present interpretation of theta activity in $\mathrm{MCI}$ patients as compensatory seems to be contradictory with the correlation found in previous studies between the increasing power of this band and the cognitive impairment in AD and MCI patients (Coben et al., 1985; Prichep et al.,

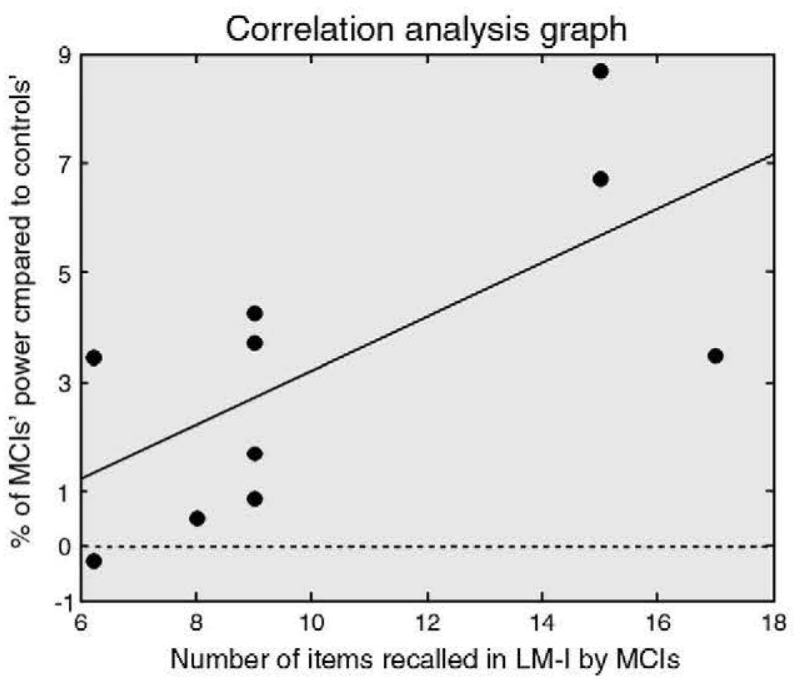

Fig. 3. This figure shows the significant correlations $(r=0.692, p<0.03)$ between the theta power changes and LM-I subtest performance. The $\mathrm{Y}$ axis shows the percentage of MCI's power compared to controls. The percentage was calculated based on the statistically significant sources and time windows of the theta band. The $\mathrm{X}$ axis shows the number of items recalled in LM-I by MCI subjects.
1994, 2006; Jelic et al., 1996, 2000; Grunwald et al., 2007; Moretti et al., 2009). However, these studies analyze brain activity during resting state conditions. Thus, in addition to the difficulty to compare differences of power along the frequency bands and in different conditions (resting state versus memory task), it is important to point that these two conditions could even show results in opposite directions (see Bajo et al. (2010) and Gómez et al. (2009)).

In studies were the oscillatory activity was recorded with EEG during the performance of a memory task, theta activity has shown a compatible profile of activity with that found in the current study. In this sense, during two n-back studies Missonnier et al. (2006a) and Deiber et al. (2009) showed a decreased theta induced and event related synchronization (ERS) in progressive $\mathrm{MCI}$ (PMCI) but not in stable $\mathrm{MCI}$ (SMCI). The fact that in these studies the reduced power increased the likelihood of progression to dementia, and that $\mathrm{MCl}$ patients who did not show the reduction were those who did not progress to dementia, reinforces the idea of linking the increased theta power with compensatory processes. Regarding the localization of the theta differences over the frontal lobe, it is interesting to point that the link between frontal theta activity and attentional processes has been consistently reported (Demiralp et al., 1994; Ishii et al., 1999; Aftanas and Golocheikine, 2001). Furthermore, its dependency on the attention level required has been established (Missonnier et al., 2006b; Deiber et al., 2007). These findings, together with the high task accuracy obtained during the recording, suggest the possible role of higher theta power in promoting the level of attention towards target stimuli that enables $\mathrm{MCI}$ patients to compensate for the cognitive decline. The fact that theta hyperactivation in $\mathrm{MCl}$ patients is concentrated within the right hemisphere indicates the necessity to recruit additional areas over the non-dominant hemisphere during a verbal task in right-handed subjects. This effect has been described 
previously as a compensatory mechanism in healthy elders, performing a verbal memory task, and has been referred to as the so-called HAROLD model (Hemispheric Asymmetry Reduction in Older adults; (Cabeza, 2002)).

Through these data, we have been able to detect an additional effort of the MCI group in the form of increased theta power which probably contributes to achieve similar level of performance than the control group (see Dickerson et al. (2005) for similar interpretation with fMRI data).

Another frequency band which showed between group differences was in the range of the beta band. MCI subjects showed lower beta band power reduction, in respect to the baseline and compared to controls, in right fronto-parietal regions at late latencies (between 645 and $801 \mathrm{~ms}$ ). Beta band desynchronization in healthy elderly has been reported during the execution of the Sternberg paradigm at the recognition stage and thus, has been considered one of the neural mechanisms necessary to perform short-term memory tasks (Karrasch et al., 2004; Krause et al., 2007). Beta band profile of activity found in the present study did not correlate with memory test performance and consequently, we are not able to interpret this finding as a compensatory mechanism. In fact and in line with our results, a recent paper by Kurimoto et al. (2011) using the Sternberg paradigm with AD patients showed lower beta event related desynchronization over the right hemisphere and interpreted this finding as a dysfunctional mechanism due to the fail of the memory networks to desynchronize in beta band.

Regarding alpha band, MCIs revealed less power than controls in the temporo-occipital region of the right hemisphere between 489 and $528 \mathrm{~ms}$. Alpha band activity has been related with an active cognitive process during memory tasks (Palva and Palva, 2007). In fact, increases in both power and functional connectivity measures have been observed as a function of memory load or during working memory (Jensen et al., 2002; Palva et al., 2010; Van Dijk et al., 2010; Meeuwissen et al., 2011; Palva and Palva, 2011). In these studies, the increasing of alpha band activity has been interpreted as a necessary mechanism to improve memory abilities.

More related to our work, the reduction in alpha power has also been observed during memory tasks and its meaning has been subjected to several interpretations. In one hand, it could reflect a lack of inhibition over task-irrelevant regions, which subsequently would affect performance (Jensen and Mazaheri, 2010). On the other hand, could be that these reduced values reflect a pathological pattern specific of MCI. In this line, Van Strien et al. (2005) observed alpha power increases during the detection of previously presented items suggesting its role in recognition processes. In addition, the reduction of posterior alpha power has been defined as the hallmark of mild AD (Babiloni et al., 2004) and as the discriminator of MCI subjects who convert to AD (Huang et al., 2000) during rest conditions. In line with these interpretations, Van der Hiele et al. (2007b) observed a decrease in alpha activity in AD patients which correlated with a worse performance on cognitive tests. Taking all these results together, we suggest that the decrease in alpha power observed in the MCI participants could be indicating a failure of the system to use a brain mechanism necessary to improve memory function.

Similarly to alpha band results, MCI subjects also showed less gamma power values than controls over left parietal regions between 372 and $489 \mathrm{~ms}$. Gamma band oscillations have been proposed to play a key role in the synchronization of the cortical networks involved in object representation, attention and memory processes (Tallon-Baudry et al., 1999; Kaiser and Lutzenberger, 2003; Daskalakis et al., 2008; Schneider et al., 2008), and modifications have been related with brain degenerative pathologies such as MCI (Stam et al., 2002; Koenig et al., 2005). Thus, this decreased in power seems to reflect a difficulty to execute the fast frequency mechanisms necessary to communicate information in the brain.

Taking together the reduction in power on high frequency bands, previous neuroimaging literature has considered brain hypoactivation as dysfunctional due to its relation to reduced task performance in $A D$ patients (Small et al., 1999; Celone et al., 2006; Han et al., 2009; Clément and Belleville, 2010). According to this interpretation, any deviant activity from the pattern shown by the control group would be considered pathological.

Therefore, based on the literature and on the fact that we did not find any correlation between cognitive performance and power in beta, alpha and gamma bands, we suggest that these patterns of activity could represent neuronal dysfunction underlying the MCI pathology during the execution of this memory task. However, further studies would be suitable to better delineate their meaning.

In sum, our results point towards a modulation of the neuronal oscillatory activity which suggests the existence of compensatory and dysfunctional mechanisms in the MCI brain. The significance of the compensation/dysfunction duality is still an open issue. Further, longitudinal studies which evaluate whether brain anatomical alterations in $\mathrm{MCl}$ or brain activity changes, as the observed in the present study, manifest stable predictors to AD are very relevant since they can offer highly useful information for diagnosis, prognosis and interventions (Bajo et al., 2012; Geroldi et al., 2006). Due to the probable transient nature of these mechanisms and their relationship to several factors, such as disease onset and its accumulating underlying neuropathology, brain/ cognitive resources, genetics and/or environmental influences, the inclusion of these factors in future studies would improve our knowledge of $\mathrm{MCl}$ and its progression to $\mathrm{AD}$ (Feldman et al., 2010).

\section{Conclusions}

To conclude, our results suggest that even in the presence of objective memory alterations, the MCl's brain is able to match control subject's memory performance by modulating theta band power in frontal regions early after target stimulus onset. It would be interesting to assess if these processes could be enhanced by cognitive training.

The present report characterized the duality of oscillatory brain responses in $\mathrm{MCl}$ subjects and aimed to overcome some of the traditional difficulties in the analysis of neuronal electromagnetic data: 1) the assumption of the stationary nature of neurophysiological signals, and 2 ) the localization of oscillatory generator sources. Additionally, future studies should evaluate: 1 ) brain oscillatory patterns along the continu$\mathrm{um}$ to $\mathrm{AD}, 2$ ) brain patterns between progressive and stable $\mathrm{MCI}$ subjects, 3) the influence of factors, such as advanced neuropathology, brain/cognitive reserve, genetic and/or environmental influences and 4) connectivity between brain regions.

\section{Financial support}

This study was partially supported by a pre-doctoral fellow of the Basque Government to S.A., the MADRI.B (CAM $\mathrm{i}+\mathrm{d}+\mathrm{I}$ project; S-SAL-0312-2006) and the Spanish Ministry of Science SEJ-2006-07560 and PSI2009-14415-C03-01.

\section{Disclosure statement}

There are no actual or potential conflicts of interest.

\section{Acknowledgments}

The authors thank P. Cuesta, D. del Río and J. García-Pacios for the helpfully discussions and technical support.

Aftanas, L.I., Golocheikine, S.A., 2001. Human anterior and frontal midline theta and lower alpha reflect emotionally positive state and internalized attention: highresolution EEG investigation of meditation. Neuroscience Letters 310, 57-60.

Alescio-Lautier, B., Michel, B.F., Herrera, C., Elahmadi, A., Chambon, C., Touzet, C., Paban, V., 2007. Visual and visuospatial short-term memory in mild cognitive impairment and Alzheimer disease: role of attention. Neuropsychologia 45, 1948-1960. 
Axmacher, N., Henseler, M.M., Jensen, O., Weinreich, I., Elger, C.E., Fell, J., 2010. Crossfrequency coupling supports multi-item working memory in the human hippocampus. Proceedings of the National Academy of Sciences of the United States of America 107, 3228-3233.

Babiloni, C., Brancucci, A., Arendt-Nielsen, L., Babiloni, F., Capotosto, P., Carducci, F., et al., 2004. Alpha event-related desynchronization preceding a go/no-go task: a highresolution EEG study. Neuropsychology 18, 719-728.

Baddeley, A., 1991. The decay of working memory in Alzheimer disease. Brain 114, 2521-2542.

Bajo, R., Maestú, F., Nevado, A., Sancho, M., Gutiérrez, R., Campo, P., et al., 2010. Functional connectivity in mild cognitive impairment during a memory task: implications for the disconnection hypothesis. Journal of Alzheimer's Disease 22, 183-193.

Bajo, R. Castellanos, N.P., Cuesta, P., Aurtenetxe, S., Garcia-Prieto, J., Gil-Gregorio, P., del-Pozo, F., Maestu, F., 2012. Differential patterns of connectivity in progressive mild cognitive impairment. Brain Connect 2, 21-24.

Belleville, S., Peretz, I., Malenfant, D., 1996. Examination of the working memory components in normal aging and in dementia of the Alzheimer type. Neuropsychologia $34,195-207$

Bokde, A.L., Karmann, M., Born, C., Teipel, S.J., Omerovic, M., Ewers, M., Frodl, T., Meisenzahl, E., Reiser, M., Möller, H.J., Hampel, H., 2010. Altered brain activation during a verbal working memory task in subjects with amnestic mild cognitive impairment. Journal of Alzheimer's Disease 21, 103-118.

Bouhamidi, A., Jbilou, K., 2007. Sylvester Tikhonov-regularization methods in image restoration. Journal of Computational and Applied Mathematics 206, 86-98.

Brookes, M.J., Gibson, A.M., Hall, S.D., Furlong, P.L., Barnes, G.R., Hillebrand, A., et al 2005. GLM-beamformer method demonstrates stationary field, alpha ERD and gamma ERS co-localisation with FMRI BOLD response in visual cortex. NeuroImage $26,302-308$.

Buzaki, G., 2006. Rythms of the Brain. Oxford University Press

Cabeza, R., 2002. Hemispheric asymmetry reduction in older adults: the HAROLD model. Psychology and Aging 17, 85-100.

Cabeza, R., Anderson, N.D., Locantore, J.K., McIntosh, A.R., 2002. Aging gracefully: compensatory brain activity in high-performing older adults. Neurolmage 17, 1394-1402

Caza, N., Belleville, S., 2008. Reduced short-term memory capacity in Alzheimet's disease: the role of phonological, lexical, and semantic processing. Memory 16, 341-350.

Celone K A Calhoun, VD Dickerson, BC Atri, A Chut, EF Miller, S. et al, 2006. Altetations in memory networks in mild cognitive impairment and Alzheimer's disease: an independent component analysis. The Journal of Neuroscience 4 (26), 10222-10231.

Clément, F., Belleville, S., 2010. Compensation and disease severity on the memoryrelated activations in mild cognitive impairment Biological Psychiatry 68, 894-902.

Coben, L.A., Danziger, W., Storandt, M., 1985. A longitudinal study of mild dementia of Alzheimer type: changes at 1 year and at 2.5 years. Electroencephalography and Clinical Neurophysiology 61, 101-112.

Collins, D.L., Zijdenbos, A.P., Kollokian, V., Sled, J.G., Kabani, N.J., Holmes, C.J., 1998 Design and construction of a realistic digital brain phantom. IEEE Transactions on Medical Imaging 17, 463-468.

Cummins, T.D., Broughton, M., Finnigan, S., 2008. Theta oscillations are affected by amnestic mild cognitive impairment and cognitive load. International Journal of Psychophysiology 70 (1), 75-81.

Daskalakis, Z.J., Farzan, F., Barr, M.S., Rusjan, P.M., Favalli, G., Levinson, A.J., et al., 2008 Evaluating the relationship between long interval cortical inhibition, working memory and gamma band activity in the dorsolateral prefrontal cortex. Clinical EEG and Neuroscience 39, 150-155.

De Toledo-Morrell, L., Evers, S., Hoeppner, T.J., Morrell, F., Garron, D.C., Fox, J.H., 1991. A 'stress' test for memory dysfunction. Electrophysiologic manifestations of early Alzheimer's disease. Archives of Neurology 48, 605-609.

Deiber, M.P., Missonnier, P., Bertrand, O., Gold, G., Fazio-Costa, L., Ibañez, V., et al., 2007. Distinction between perceptual and attentional processing in working memory tasks: a study of phase-locked and induced oscillatory brain dynamics. Journal of Cognitive Neuroscience 19,158-172.

Deiber, M.P., Ibañez, V., Missonnier, P., Herrmann, F., Fazio-Costa, L., Gold, G., et al 2009. Abnormal-induced theta activity supports early directed-attention network deficits in progressive MCI. Neurobiology of Aging 30, 1444-1452.

Demiralp, T., Basar-Eroglu, C., Rahn, E., Basar, E., 1994. Event-related theta thythms in cat hippocampus and prefrontal cortex during an omitted stimulus paradigm. International Joumal of Psychophysiology 18, 35-48.

Dickerson, B.C., Sperling, R.A., 2008. Functional abnormalities of the medial tempora lobe memory system in mild cognitive impairment and Alzheimer's disease: insights from functional MRI studies. Neuropsychologia 46, 1624-1635.

Dickerson, B.C., Salat, D.H., Greve, D.N., Chua, E.F., Rand-Giovannetti, E., Rentz, D.M., et al. 2005. Increased hippocampal activation in mild cognitive impairment compared to normal aging and $\mathrm{AD}$. Neurology 9 (65), 404-411.

Feldman, H.H., Jacova, C., Cummings, J.L., Dekosky, S.T., Barberger-Gateau, P. Delacourte, A., et al., 2010. Revising the definition of Alzheimer's disease: a new lexicon. Lancet Neurology 9, 1118-1127.

Fell, J., Klaver, P., Elfadil, H., Schaller, C., Elger, C.E., Fernández, G., 2003. Rhinal-hippocampa theta coherence during declarative memory formation: interaction with gamma synchronization? The European Joumal of Neuroscience 17, 1082-1088.

Finnigan, S., Robertson, I.H., 2011. Resting EEG theta power correlates with cognitive performance in healthy older adults. Psychophysiology 48, 1083-1087.

Friston, K.J., Price, C.J., 2003. Degeneracy and redundancy in cognitive anatomy. Trends in Cognitive Sciences 7, 151-152.

Geroldi, C., Rossi, R., Calvagna, C., Testa, C., Bresciani, L., Binetti, G., Zanetti, O., Frisoni, G.B., 2006. Medial temporal atrophy but not memory deficit predicts progression to dementia in patients with mild cognitive impairment. Journal of Neurology, Neurosurgery, and Psychiatry 77, 1219-1222.
Giannakopoulos, P., Missonnier, P., Kövari, E., Gold, G., Michon, A., 2009. Electrophysiological markers of rapid cognitive decline in mild cognitive impairment. Frontiers of Neurology and Neuroscience 24, 39-46.

Gómez, C., Stam, C.]., Hornero, R., Fernández, A., Maestú, F., 2009. Disturbed beta band functional connectivity in patients with mild cognitive impairment: an MEG study. IEEE Transactions on Biomedical Engineering 56 (6), 1683-1690 (Jun).

Grady, C.L., McIntosh, A.R., Craik, F.I., 2005. Task-related activity in prefrontal cortex and its relation to recognition memory performance in young and old adults. Neuropsychologia 43, 1466-1481.

Grunwald, M., Busse, F., Hensel, A., Riedel-Heller, S., Kruggel, F., Arendt, T., et al., 2002. Theta-power differences in patients with mild cognitive impairment under rest condition and during haptic tasks. Alzheimer Disease and Associated Disorders $16,40-48$.

Grunwald, M., Hensel, A., Wolf, H., Weiss, T., Gertz, H.J., 2007. Does the hippocampal atrophy correlate with the cortical theta power in elderly subjects with a range of cognitive impairment? Journal of Clinical Neurophysiology 24, 22-26.

Gurtubay, I.G., Alegre, M., Labarga, A., Malanda, A., Iriarte, J., Artieda, J., 2001. Gamma band activity in an auditory oddball paradigm studied with the wavelet transform. Clinical Neurophysiology 112, 1219-1228.

Han, S.D., Bangen, K.J., Bondi, M.W., 2009. Functional magnetic resonance imaging of compensatory neural recruitment in aging and risk for Alzheimer's disease: review and recommendations. Dementia and Geriatric Cognitive Disorders 27, 1-10.

Hogan, M.J., Swanwick, G.R., Kaiser, J., Rowan, M., Lawlor, B., 2003. Memory-related EEG power and coherence reductions in mild Alzheimer's disease. International Journal of Psychophysiology 2003 (49), 147-163.

Huang, M.X., Mosher, J.C., Leahy, R.M., 1999. A sensor-weighted overlapping-sphere head model and exhaustive head model comparison for MEG. Physics in Medicine and Biology 44, 423-440.

Huang, C. Wahlund, L., Dierks, T., Julin, P., Winblad, B., Jelic, V., 2000. Discrimination of Alzheimer's disease and mild cognitive impairment by equivalent EEG sources: a cross-sectional and longitudinal study. Clinical Neurophysiology 111, 1961-1967.

Ishii, R., Shinosaki, K., Ukai, S., Inouye, T., Ishihara, T., Yoshimine, T., Hirabuki, N.. Asada, H., Kihara, T., Robinson, S.E., Takeda, M., 1999. Medial prefrontal cortex generates frontal midline theta thythm. Neuroreport 10, 675-679

Jelic, V., Shigeta, M., Julin, P., Almkvist, O., Winblad, B., Wahlund, L.O., 1996. Quantitative electroencephalography power and coherence in Alzheimer's disease and mild cognitive impairment. Dementia 7, 314-323.

Jelic, V., Johansson, S.E., Almkvist, O., Shigeta, M., Julin, P., Nordberg, A., et al., 2000. Quantitative electroencephalography in mild cognitive impairment: longitudinal changes and possible prediction of Alzheimer's disease. Neurobiology of Aging $21,533-540$.

Jensen, O., Mazaheri, A., 2010. Shaping functional architecture by oscillatory alpha activity: gating by inhibition. Frontiers in Human Neuroscience 4 (4), 186.

Jensen, O., Tesche, C.D., 2002. Frontal theta activity in humans increases with memory load in a working memory task. The European Joumal of Neuroscience 15, 1395-1399.

Jensen, O., Vanni, S., 2002. A new method to identify multiple sources of oscillatory activity from magnetoencephalographic data. Neurolmage $15,568-574$

Jensen, O., Gelfand, J., Kounios, J., Lisman, J.E., 2002. Oscillations in the alpha band $(9-12 \mathrm{~Hz})$ increase with memory load during retention in a short-term memory task. Cerebral Cortex 12, 877-882.

Jiang, Z.Y., 2005. Study on EEG power and coherence in patients with mild cognitive impairment during working memory task. Journal of Zhejiang University. Science. B 6, 1213-1219.

Johnson, K.A., Fox, N.C., Sperling, R.A., Klunk, W.E., 2012. Brain imaging in Alzheimer disease. Cold Spring Harbor Perspectives in Medicine 2, a006213

Kaiser, J., Lutzenberger, W., 2003. Induced gamma-band activity and human brain function. The Neuroscientist 9, 475-484.

Karrasch, M., Laine, M., Rapinoja, P., Krause, C.M., 2004. Effects of normal aging on event-related desynchronization/synchronization during a memory task in humans. Neuroscience Letters 366, 18-23.

Karrasch, M., Laine, M., Rinne, J.O., Rapinoja, P., Sinervä, E., Krause, C.M., 2006. Brain oscillatory responses to an auditory-verbal working memory task in mild cognitive impaitment and Alzheimer's disease. International Journal of Psychophysiology 59, 168-178.

Klimesch, W., 1996. Memory processes, brain oscillations and EEG synchronization. International Journal of Psychophysiology 24, 61-100.

Klimesch, W., 1999. EEG alpha and theta oscillations reflect cognitive and memory performance: a review and analysis. Brain Research Reviews 29, 169-195.

Klimesch, W., Doppelmayr, M., Russegger, H., Pachinger, T., Schwaiger, ]., 1998. Induced alpha band power changes in the human EEG and attention. Neuroscience Letters 244 (2), 73-76 (Mar 13)

Kochan, N.A., Breakspear, M., Slavin, M.J., Valenzuela, M. McCraw, S., Brodaty, H. Sachdev, P.S., 2010. Functional alterations in brain activation and deactivation in mild cognitive impairment in response to a graded working memory challenge. Dementia and Geriatric Cognitive Disorders 30, 553-568.

Koenig, T., Prichep, L., Dierks, T., Hubl, D., Wahlund, L.O., John, E.R., et al., 2005. Decreased EEG synchronization in Alzheimer's disease and mild cognitive impairment. Neurobiology of Aging 26, 165-171.

Krause, C.M., Pesonen, M., Hämäläinen, H., 2007. Brain oscillatory responses during the different stages of an auditory memory search task in children. Neuroreport 18, 213-216.

Kurimoto, R., Ishii, R., Canuet, L., Ikezawa, K., Iwase, M., Azechi, M., et al., 2011. Induced oscillatory responses during the Sternberg's visual memory task in patients with Alzheimer's disease and mild cognitive impairment. Neurolmage http://dx.doi.org/ 10.1016/.neuroimage.2011.10.061

Leiberg, S., Lutzenberger, W., Kaiser, J., 2006. Effects of memory load on cortical oscillatory activity during auditory pattern working memory. Brain Research 1120 , 131-140. 
Long, X., Zhang, L., Liao, W., Jiang, C., Qiu, B., The Alzheimer's Disease Neuroimaging Initiative, 2012. Distinct laterality alterations distinguish mild cognitive impairment and Alzheimer's disease from healthy aging: Statistical parametric mapping with high resolution MRI. Human Brain Mapping http://dx.doi.org/10.1002/hbm.22157 (Aug 28).

Maestû, F., Femández, A., Simos, P.G., Gil-Gregorio, P., Amo, C., Rodriguez, R., et al., 2001. Spatio-temporal patterns of brain magnetic activity during a memory task in Alzheimer's disease. Neuroreport 12, 3917-3922.

Maestú, F., Campo, P., Del Río, D., Moratti, S., Gil-Gregorio, P., Fernández, A., et al., 2008. Increased biomagnetic activity in the ventral pathway in mild cognitive impairment. Clinical Neurophysiology 119, 1320-1327.

Mallat, S., 1998. A Wavelet Tour of Signal Processing. Academic Press, San Diego.

Meeuwissen, E.B., Takashima, A., Fernández, G., Jensen, O., 2011. Increase in posterior alpha activity during rehearsal predicts successful long-term memory formation of word sequences. Human Brain Mapping 32, 2045-2053.

Missonnier, P., Gold, G., Herrmann, F.R., Fazio-Costa, L., Michel, J.P., Deiber, M.P., et al., 2006a. Decreased theta event-related synchronization during working memory activation is associated with progressive mild cognitive impairment. Dementia and Geriatric Cognitive Disorders 22, 250-259.

Missonnier, P., Deiber, M.P., Gold, G., Millet, P., Gex-Fabry Pun, M., Fazio-Costa, L., et al., 2006b. Frontal theta event-related synchronization: comparison of directed attention and working memory load effects. Joumal of Neural Transmission 113, 1477-1486.

Moratti, S., Rubio, G., Campo, P., Keil, A., Ortiz, T., 2008. Hypofunction of right temporoparietal cortex during emotional arousal in depression. Archives of General Psychiatry 65, 532-541.

Moretti, D.V., Fracassi, C., Pievani, M., Geroldi, C., Binetti, G., Zanetti, O., et al., 2009. Increase of theta/gamma ratio is associated with memory impairment. Clinical Neurophysiology 120, 295-303.

Neuper, C., Pfurtscheller, G., 2001. Evidence for distinct beta resonance frequencies in human EEG related to specific sensorimotor cortical areas. Clinical Neurophysiology $112,2084-2097$.

Oldfield, R.C., 1971. The assessment and analysis of handedness: the Edinburgh inventory. Neuropsychologia 9, 97-113.

Osipova, D., Ahveninen, J., Jensen, O., Ylikoski, A., Pekkonen, E., 2005. Altered generation of spontaneous oscillations in Alzheimer's disease. Neurolmage 27, 835-841.

Palva, S., Palva, J.M., 2007. New vistas for alpha-frequency band oscillations. Trends in Neurosciences $30,150-158$.

Palva, S., Palva, J.M., 2011. Functional roles of alpha-band phase synchronization in local and large-scale cortical networks. Frontiers in Psychology 2, 204.

Palva, J.M., Monto, S., Kulashekhar, S., Palva, S., 2010. Neuronal synchrony reveals working memory networks and predicts individual memory capacity. Proceedings of the National Academy of Sciences of the United States of America 107, $7580-7585$.

Petersen, R.C., 2004. Mild cognitive impairment as a diagnostic entity. Journal of Internal Medicine 256, 183-194.

Petersen, R.C., Doody, R., Kurz, A., Mohs, R.C., Morris, J.C., Rabins, P.V., y Winblad, B., 2001. Current concepts in mild cognitive impairment. Archives of Neurology 58 , 1985-1992.

Petersen, R.C., Parisi, J.E., Dickson, D.W., Johnson, K.A., Knopman, D.S., Boeve, B.F. yKokmen, E., 2006. Neuropathologic features of amnestic mild cognitive impairment. Archives of Neurology 63, 665-672.

Pfeffer, R.I., Kurosaki, T.T., Harrah Jr., C.H., Chance, J.M., Filos, S., 1982. Measurement of functional activities in older adults in the community. Journal of Gerontology 37 , 323-329.
Pivik, RT., Broughton, R.., Coppola, R., Davidson, RJ., Fox, N., Nuwer, M.R., 1993. Guidelines for the recording and quantitative analysis of electroencephalographic activity in research contexts. Psychophysiology 30, 547-558.

Prichep, L.S., John, E.R., Ferris, S.H., Reisberg, B., Almas, M., Alper, K., et al., 1994. Quantitative EEG correlates of cognitive deterioration in the elderly. Neurobiology of Aging 15, 85-90.

Prichep, L.S., John, E.R., Ferris, S.H., Rausch, L., Fang, Z., Cancro, R., et al., 2006. Prediction of longitudinal cognitive decline in normal elderly with subjective complaints using electrophysiological imaging. Neurobiology of Aging 27, 471-481.

Scarmeas, N., Zarahn, E., Anderson, K.E., Hilton, J., Flynn, J., Van Heertum, R.L., et al., 2003. Cognitive reserve modulates functional brain responses during memory tasks: a PET study in healthy young and elderly subjects. Neurolmage 19, 1215-1227.

Schneider, T.R., Debener, S., Oostenveld, R., Engel, A.K., 2008. Enhanced EEG gammaband activity reflects multisensory semantic matching in visual-to-auditory object priming. Neurolmage 1 (42), 1244-1254.

Small, S.A., Perera, G.M., DeLaPaz, R., Mayeux, R., Stern, Y., 1999. Differential regiona dysfunction of the hippocampal formation among elderly with memory decline and Alzheimer's disease. Annals of Neurology 45, 466-472.

Stam, C.J., van Cappellen van Walsum, A.M., Pijnenburg, Y.A., Berendse, H.W., de Munck, J.C., Scheltens, P., et al., 2002. Generalized synchronization of MEG recordings in Alzheimer's disease: evidence for involvement of the gamma band. Journal of Clinical Neurophysiology 19, 562-574.

Stam, C.J., Jone, B.F., Manshanden, I., van Cappellen van Walsum, A.M., Montez, T. Verbunt, J.P., de Muck, J.C., van Dijk, B.W., Berendse, H.W., Scheltens, P., 2006. Magnetoencephalographic evaluations of resting-state functional connectivity in Alzheimer's disease. Neuroimage 32, 1335-1344.

Tallon-Baudry, C., Bertrand, O., Delpuech, C., Permier, J., 1997. Oscillatory gamma-band $(30-70 \mathrm{~Hz})$ activity induced by a visual search task in humans. The Journal of Neuroscience $17,722-734$.

Tallon-Baudry, C., Kreiter, A., Bertrand, O., 1999. Sustained and transient oscillatory responses in the gamma and beta bands in a visual short-term memory task in humans. Visual Neuroscience $16,449-459$

Twamley, E.W., Ropacki, S.A., Bondi, M.W., 2006. Neuropsychological and neuroimaging changes in preclinical Alzheimer's disease. Journal of the International Neuropsychological Society $12,707-735$.

Van der Hiele, K. Vein, AA, Kramer, C.G Reijntjes, RH van Buchem, MA Westendorp, R.G., et al., 2007a. Memory activation enhances EEG abnormality in mild cognitive impairment. Neurobiology of Aging 28, 85-90.

Van der Hiele, K., Vein, A.A., Reijntjes, R.H., Westendorp. R.G., Bollen, E.L., van Buchem, M.A., et al., 2007b. EEG correlates in the spectrum of cognitive decline. Clinical Neurophysiology 118, 1931-1939.

Van Dijk, H., Nieuwenhuis, I.L., Jensen, O., 2010. Left temporal alpha band activity increases during working memory retention of pitches. The European Journal of Neuroscience $31,1701-1707$.

Van Strien, J.W., Hagenbeek, R.E., Stam, C.J., Rombouts, S.A., Barkhof, F., 2005. Changes in brain electrical activity during extended continuous word recognition. NeuroImage 26, 952-959.

Zheng, L. Jiang, Z., Yu, E., 2007. Alpha spectral power and coherente in the patients with mild cognitive impairment during a three-level working memory task. Joumal of Zhejiang University. Science 8,584-592. 